\title{
EDUCACIÓN Y MISERICORDIA
}

\section{Mons. Enrico dal Covolo, S. D. B.* Pontificia Universidad Lateranense de Roma}

Resumen: El presente texto nos acercará a la mirada que Mons. Enrico dal Covolo sostiene sobre la educación. Asimismo, con motivo del Jubileo de la Misericordia llevado a cabo entre el 2015 y el 2016, nos hablará de los alcances que este don, el cual Dios provee, puede tener en el campo educativo. Al dirigirse principalmente a los jóvenes estudiantes, el autor apelará a un lenguaje llano y natural con el que intentará adentrarlos en el potencial que puede ofrecer la educación, relacionada con la misericordia, en cuanto a un cambio en la perspectiva que las personas tienen de la realidad y del prójimo. Para sostener esta postura, entre otras fuentes, Mons. Dal Covolo empleará diversos documentos en los que el papa Francisco ha hecho énfasis en su predicación sobre la misericordia, sin dejar de lado lo educativo. Finalmente, gracias a este impulso dado por el Santo Padre,

* Enrico dal Covolo, S. D. B. es obispo titular de Eraclea y magnífico rector de la Pontificia Universidad Lateranense. Se graduó en estudios clásicos en la Universidad Católica del Sagrado Corazón de Milán. Asimismo, completó sus estudios en el Departamento de Estudios Religiosos de la misma universidad y en el Instituto Patrístico Agustiniano de Roma, donde obtuvo un doctorado en Teología y Ciencia Patrística. En 2002, Juan Pablo II lo nombró consultor de la Congregación para la Doctrina de la Fe. Luego, en el 2008, Benedicto XVI lo nombró consultor de la Congregación para el Clero. En 2009, fue nombrado miembro de la Pontificia Comisión de Arqueología Sacra. El 30 de junio de 2010, Benedicto XVI lo nombró 
el autor brindará las bases de lo que ha tenido a bien catalogar como una pedagogía de la misericordia.

Palabras Clave: Francisco, misericordia, educación, jóvenes, humanidad.

\section{EDUCATION AND MERCY}

ABSTRACT: The current paper is a way of approaching to Monsignor Enrico dal Covolo's view in relation to education. Similarly, for the sake of Jubilee of Mercy, carried out between 2015 and 2016, he will talk about the outcomes this coming from God gift can have in education. Addressing specially to young students, the author will apply a simple and natural language through which he will attempt to introduce them in the possibilities education related to Mercy can provide. This means, the change in the perspective

rector de la Pontificia Universidad Lateranense de Roma, y el 15 de septiembre del mismo año fue elevado a la dignidad episcopal, asignándosele la sede titular de Eraclea. Por otro lado, dirige la Colección Caridad Pastoral, de la Editorial Rogate, para la formación permanente de los sacerdotes y consagrados. Ha dedicado, también, numerosas publicaciones sobre los Padres de la Iglesia y sobre las relaciones entre las instituciones sociopolíticas y la estructura de la Iglesia antigua, entre las cuales figuran los siguientes: Los Severos y el Cristianismo; Iglesia y Sociedad Política. Áreas de la laicidad en el cristianismo de los origenes; Los Padres de la Iglesia, maestros de formación sacerdotal; y Formas de vida espiritual en los Padres de la Iglesia. Ha publicado, además, contribuciones y misceláneas de estudio sobre algunos documentos del magisterio eclesiástico, los cuales se mencionan a continuación: la Encíclica Redemptoris Mater, La instrucción sobre el estudio de los Padres de la Iglesia en la formación Sacerdotal; la Encíclica Redemptoris Missio; la Exhortación Apostólica Pastores Dabo Vobis; el Catecismo de la Iglesia Católica; la Encíclica Fides et ratio; la Bula de la Convocación del Gran Jubileo del año 2000; la Encíclica Ecclesia de Eucharistia; el Compendio del Catecismo de la Iglesia Católica; la Encíclica Deus caritas est, la Encíclica Caritas in veritate y la Encíclica Lumen Fidei. 
people have about reality and their fellows. In order to demonstrate his posture, Monsignor Dal Covolo uses among many resources, a set of diverse documents in which Pope Francis has made emphasis in the preaching about Mercy without neglecting the educational aspect. Finally, thanks to the Holy Father's impulse, the author will provide the basis of which he has entitled The pedagogy of Mercy.

KeYwOrDs: Francis, mercy, education, young people, humanity.

\section{La Educación como Apertura a la Realidad}

1 dirigirme a vosotros, queridos estudiantes, recuerdo muy bien
cuando, a mi vez, yo me encontraba en vuestra misma condición
de estudiante. La juventud es la etapa de la vida que expresa la mayor energía biológica y la máxima vitalidad física, pero, al mismo tiempo, se ve acosada por angustias, debilidades e inseguridades. Como ya observó Erik Erikson, en ninguna otra etapa del ciclo de la vida la esperanza de encontrarse —así como la amenaza de perderse- están tan íntimamente relacionadas. Son necesarios valor y determinación, pero, a la vez, se requiere afinar una mirada de misericordia, en primer lugar, hacia nosotros mismos.

Para ayudaros a pensar acerca de lo que os acabo de decir, os pido que hagáis un pequeño ejercicio con la imaginación. Este, personalmente, me ha ayudado para ir más allá de mis fuerzas, en la lucha contra los demonios del juicio y de la soberbia, los demonios que acechan cuando se estudia y se aumenta la propia cultura. Imaginaos dibujar un cuadrado delante de vosotros. Quien tenga lápiz y papel puede hacerlo en una hoja. ¿Qué hay dentro de este cuadrado? Mi mundo ideal: mi yo ideal, mi ideal 
de familia, el trabajo, la parroquia, la chica o chico, el cristiano, el feligrés, el profesor, el ideal superior. ¿Cuál es el peligro? Es tener la tentación de ver el mundo a través de esa ventana.

La consecuencia inevitable es la siguiente: todo lo que está fuera, que es real, es juzgado y vivido de forma predeterminada, que es negativo con respecto a ese ideal, que es la medida de mi evaluación y mi percepción. Pero nosotros no vivimos allí, en ese cuadrado. ¡No vivimos allí dentro, vivimos en la realidad! Y donde vivo, la gente, las relaciones y situaciones no reflejarán nunca mi ideal. Y, a Dios gracias, ¡el mundo no es a imagen y semejanza de nuestras propias proyecciones humanas! Pero querer vivir en ese cuadrado significa renunciar a las personas y las relaciones, a las propias ideas, principios y convicciones. El papa Francisco, en aquel tiempo el cardenal Bergoglio, llamó a estas ideas "nominalismos": "Los nominalismos no convocan nunca. A lo sumo, clasifican, citan, definen, pero no convocan. Lo que convoca es la realidad iluminada por el razonamiento, por la idea, @ por la captación intuitiva por parte de ellos” (Bergoglio, 2010, párr. 77). Existe el peligro de que este cuadrado sea un lugar inhumano, implacable, en primer lugar, hacia nosotros mismos (si seguimos el yo ideal), y luego hacia los demás.

Pensemos ahora en Jesús. Estaba con todos aquellos que se encontraban fuera de este cuadrado ideal, con aquellos que los sabios de su tiempo juzgaban negativamente: la hemorroísa, la samaritana, Zaqueo, Mateo, el leproso, el endemoniado, Bartimeo ... "Jesús no ve la realidad desde el exterior, sin permanecer indiferente, como si hiciese una foto, sino que se involucra" (Francisco, 2016a, p.102). Efectivamente, va en busca de esas semillas de la verdad y la belleza que están presentes en cada realidad que, en sí mismas, son imperfectas. 
Si nos amparamos en esta perspectiva, ¡la educación tiene que ser, ante todo, un medio para abrirse a la realidad! El papa Francisco dijo:

Ésta es la primera razón por la que me encanta la escuela. Amo la escuela porque es sinónimo de apertura a la realidad. ¡ $\mathrm{Al}$ menos así tendría que ser! Ir a la escuela significa abrir la mente y el corazón a la realidad, a la riqueza de sus aspectos, de sus dimensiones. ¡Y nosotros no tenemos derecho de tener miedo de la realidad! La escuela nos enseña a entender la realidad. Ir a la escuela significa abrir la mente y el corazón a la realidad, en la riqueza de sus aspectos, de sus dimensiones. (2014, párr. 3)

Del mismo modo, una verdadera educación nos permite superar la tentación que el papa Francisco califica como "la espiritualidad del espejismo":

Caminar a través de los desiertos de la humanidad sin ver lo que realmente hay, sino lo que a nosotros nos gustaría ver; somos capaces de construir visiones del mundo, pero no aceptamos lo que el Señor pone delante de nuestros ojos. Una fe que no sabe radicarse en la vida de la gente permanece árida y, en lugar de oasis, crea otros desiertos. (2015a, párr. 7)

No obstante, esta apertura a la realidad, al liberarnos de los cierres de los nominalismos, nos permite dar una mirada de misericordia a nuestra realidad personal y a la que nos rodea. 


\section{Una Educación que habla al Corazón de la Humanidad}

Tenemos que preguntarnos, para entender completamente lo que provocó al papa Francisco a celebrar este Jubileo Extraordinario, por qué la humanidad necesita clemencia. Ante lo que voy a deciros, quedará claro cómo la educación y las instituciones relacionadas juegan un papel importante respecto a aquella. Verán, también, cómo se les pide que replanteen la situacion al compararse con este mensaje.

La mirada de Francisco tiene ante sí una humanidad herida, marcada por heridas profundas, con frecuencia sin ninguna experiencia de misericordia, tanto como a obligarla a no creer que pueda existir una redención, una curación (2016a). Sin embargo, “¡Dios no es indiferente! Dios se preocupa por la humanidad, Dios no la abandona” (2016b, párr.1). Porque Dios tiene confianza en el hombre, así como el papa Francisco. Este es uno de los elementos que caracterizan su enseńanza: una perspectiva positiva sobre el hombre, la capacidad de ser capaz de confiar en él. Pero, para ello, es necesario apuntar al corazón del hombre, incluso antes que en proyectos o diversas obras de ingeniería pastoral.

De esta frustración parece que se deriva una enorme desesperación. El mundo y las instituciones que lo gestionan, todas ellas, incluso las docentes, se concentran en un espíritu positivo para definir el "qué" y el "cómo" de la realidad, dejando de lado el "por qué". Que un joven de vuestra edad, que ha nacido en un país europeo u occidental, decida inmolarse en nombre de una ideología, causando la muerte de sus compañeros, ¿no se deduce, quizás, esta ineptitud de las instituciones y la cultura para ser capaz de responder a una necesidad más profunda, que vas más allá del simple derecho a la ciudadanía? Alcanzar un sentido es algo más que la adquisición 
de un derecho, de un reconocimiento. Se puede luchar contra la injusticia, pero mucho más difícil es sobrevivir sin ser capaz de dar un significado más profundo a la vida. Al respecto, el psiquiatra austríaco Vicktor Frankl — que sobrevivió a Auschwitz, donde perdió a su padre, su madre, su hermano y su mujer embarazada - recordaba una frase de Nietszche en El hombre en busca de sentido: "Quien tiene un 'por qué' para vivir puede soportar casi cualquier 'cómo"' (1991, p. 1).

Para este caso, se vuelven más pertinentes las palabras del Santo Padre:

Éste es el tiempo de la misericordia. La Iglesia muestra su rostro materno, su rostro de madre, a la humanidad herida. No espera a que los heridos llamen a su puerta, sino que va a buscarlos a las calles, los recoge, los abraza, los cura, hace que se sientan amados. (Francisco, 2016a, p. 12)

Es dentro de esta visión que surge la imagen de la Iglesia como un hospital de campaña:

Veo con claridad que lo que la Iglesia necesita con mayor urgencia hoy es una capacidad de curar heridas y dar calor a los corazones de los fieles, cercanía, proximidad. Veo a la Iglesia como un hospital de campaña después de una batalla. ¡Qué inútil es preguntarle a un herido si tiene altos el colesterol y el azúcar! Hay que curarle las heridas. Después podremos hablar de todo lo demás. Curar las heridas, curar las heridas... Y hay que empezar desde abajo. (Spadaro, 2013, p. 13) 
Ahora bien, dicho hospital de campaña tiene propiedades únicas que pueden y deben guiar cualquier reforma de nuestras instituciones. Estas son las que siguen:

- Es inclusivo: no rechaza a nadie;

- Está cerca de donde hay heridos;

- Es flexible, no es estático, fácil de transportar, para moverse donde hay nuevas situaciones de necesidad;

- Está descentralizado: cada unidad está preparada y es responsable, capaz de tomar la iniciativa.

Cada institución educativa tiene que inspirarse en un hospital de campaña y encontrar una referencia para su acción de promoción humana y espiritual en las obras de misericordia. Cabe ańadir que dichas obras de misericordia "nos recuerdan que nuestra fe se traduce en gestos concretos o y diarios, destinados a ayudar a nuestro prójimo en el cuerpo y el espíritu, y sobre los que seremos juzgados: nutrirlo, visitarlo, consolarlo y educarlo" (Francisco, 2016c, párr. 6). Es necesario que haya acciones concretas y visibles que muestren la cercanía de Dios a la humanidad. "El amor, después de todo, nunca podría ser una palabra abstracta. Por su misma naturaleza es la vida concreta: intenciones, actitudes, comportamientos que se verifican en el vivir cotidiano" (Francisco, 2015b, párr. 19). Esto determina la credibilidad de nuestro trabajo pastoral y educativo, y la eficacia para llegar al corazón del ser humano. 


\section{Una Educación que humaniza}

Un proyecto educativo que tenga como único objetivo transmitir el contenido no es inclusivo. "La verdadera escuela tiene que enseñar conceptos, costumbres y valores; y cuando una escuela no es capaz de hacer todo esto junto, esta escuela es selectiva, exclusiva, es para pocos" (Francisco, 2015c, párr. 4). Es la misma preocupación que Francisco presenta en la Encíclica Laudato si (LS): "La existencia de leyes y normas no es suficiente a largo plazo para limitar los malos comportamientos, aun cuando exista un control efectivo" (211), así como no es suficiente instar en el plano moral, que es bueno hacer algo en beneficio de otros (208). En primer lugar, para el Pontífice, existe la necesidad de llenar los corazones de la gente porque, según lo declarado por Benedicto XVI, los desiertos exteriores son el resultado del aumento de los desiertos interiores. En una época de crisis de sentido y de "vacío existencial" la educación no solo puede continuar con "transmitir conocimientos", sino que también tiene que asumir la tarea de "afinar la conciencia".

Hablar sobre educación es hablar de la humanidad. Más aún, ello se plasma en la labor que inspira la Iglesia:

Educar cristianamente es llevar adelante a los jóvenes, a los niños en los valores humanos en todas las realidades y una de estas realidades es lo trascendente. Para mí, la mayor crisis de la educación, en la perspectiva cristiana, es este cierre a la trascendencia. Estamos cerrados a la trascendencia. Hay que preparar los corazones para que el Seńor se manifieste, pero en la totalidad. (Francisco, 2015c, párr. 1) 
Nos encontramos en la raíz del problema de la educación. Estamos ante una actitud fundamental que el papa Francisco define en la capacidad de trascenderse a sí mismo, al salir de nosotros mismos para ir hacia el otro, ya sea una persona o la naturaleza: "Sin ella no se reconoce a las demás criaturas en su propio valor, no hay capacidad de ponerse límites para evitar el sufrimiento o el deterioro de lo que nos rodea" (LS 208). El objetivo educativo se convierte, entonces, en el estímulo de la capacidad de la empatía universal en el individuo, y esto es posible a partir de la certeza antropológica que el ser humano es capaz de degradarse, pero también de elegir lo bueno para regenerarse (205).

Reeducar a los jóvenes en la trascendencia es el verdadero antídoto contra el narcisismo rampante en las nuevas generaciones. Hay que guiar a los jóvenes para salir de sí mismos (para existir, es decir, a estar fuera, a emerger) en el sentido del mundo y de los demás, para darse a sí mismos por una causa, para establecer metas y objetivos. Con ello, ejercerán, de manera gradual, la libertad y la responsabilidad que son las claves para una verdadera existencia.

Una educación capaz de cuidar de la humanidad tiene que ser capaz de utilizar tres lenguajes, según el papa Francisco: el lenguaje de la cabeza, el lenguaje del corazón y el lenguaje de las manos. "La educación tiene que ir por estos tres caminos. Enseñar a pensar, ayudar a sentir bien y acompañar en el quehacer, es decir, que los tres lenguajes estén en armonía”. (2015c, párr. 4). Para lograr todo esto, la educación informal tiene un papel decisivo: si la educación formal se basa en un tecnicismo intelectualista y en el lenguaje de la cabeza, se empobrece; es necesario recuperar los valores del arte, del deporte, de lo libre... Entonces se trata de experimentar nuevos modelos, abrir nuevos horizontes educativos, que impulsen a toda la persona a un crecimiento y un desarrollo integrado. 


\section{A Vosotros, Estudiantes: de los Ídolos del Saber a la Misericordia}

Hice la introducción de este texto pidiéndoos que pensaseis sobre un primer riesgo, que consiste en juzgar la realidad a partir de un ideal o, en palabras del papa Francisco, desde el centro. Esto es, sin querer ensuciarse los zapatos en las periferias geográficas y existenciales de nuestra vida. Asimismo, como estudiantes, podéis correr un riesgo posterior, a saber, el de ser víctimas de los ídolos del conocimiento. Veámoslos un poco más detenidamente.

\section{- El idolo del orgullo}

Ser misericordiosos, en primer lugar, significa sufrir la propia pobreza, los propios límites y la fragilidad. "El pobre más miserable es quien no acepta reconocerse como tal. Cree que es rico, pero en realidad es el más pobre de los pobres" (Francisco, 2016c, párr. 7). Es probable que el pecado del orgullo nos arrastre a utilizar la riqueza y el poder no para servir a Dios y a los demás, sino para cegarnos y perder de vista la propia medida. Es el camino que lleva a la corrupción: corrupto es aquel que se siente sin la necesidad de perdón y misericordia, pero se justifica a sí mismo y su comportamiento; es aquel que peca y no se arrepiente, aquel que peca y pretende ser un cristiano; el corrupto es aquel que tiene una doble vida, es un verdadero hábito mental, una forma de vida. Ante esto, “Pecadores, sí, pero no corruptos!” (Francisco, 2016a, p. 87). 


\section{- El idolo de la Ley}

Es uno de los que toman la "psicología de los doctores de la Ley y de los fariseos": los cristianos que con su forma de actuar apagan lo que el Espíritu Santo enciende en el corazón de un pecador, de alguien que está en la puerta. A menudo, tienen una adhesión formal a la Ley, pero en realidad esconden heridas muy profundas. En el origen de estas actitudes está la pérdida del estupor frente a la salvación que nos fue dada. Cuando uno se siente un poco más seguro, empieza a acapararse facultades que no son suyas, sino del Señor. El estupor empieza a degradarse; esto se encuentra en la base del clericalismo o la actitud de aquellos que se sienten puros. La adhesión formal a las leyes, a nuestros esquemas de pensamiento, prevalece. Permanecemos cerrados a las sorpresas de Dios. Es "la degradación del asombro" (Francisco, 2016a, p. 82), para seguir utilizando el lenguaje del Santo Padre. Esperemos que en estos casos solo se haga un buen "resbalón" para encontrar un equilibrio saludable, tener una visión más misericordiosa de la realidad.

\section{- El idolo de la autonomía}

Consiste en ser percibidos como el resultado de las propias elecciones, como el producto de un proceso de autoconstrucción en el que el propio individuo existe para investigar y determinar quién en última instancia tendrá que ser/querrá ser. Es un mito, porque absolutiza la decisión como fuente de identidad, sin tener en cuenta cuánto se recibe de las generaciones anteriores. Un mito peligroso, pues puede crear ansiedad cuando la realización de su propia vida, 
con sus propios esfuerzos, es un fracaso. Es aquí donde puede surgir el deseo latente de renunciar a la propia autonomía, uniéndose a otros, a los que encomendar la definición de la identidad, o en busca de "soluciones mágicas" a los propios problemas. De ahí la incapacidad para abrirse a la gracia de la misericordia del Padre, que no limita nuestra libertad, sino, por el contrario, la aumenta.

\section{- El idolo del bienestar interior}

Es una búsqueda de espiritualidad que se esconde detrás de una necesidad más general de bienestar psicofísico o psicorreligioso. La fe cristiana tiene, sobre todo, un carácter de apertura a los demás y al mundo, mientras que la búsqueda de "bienestar espiritual" tiene muy a menudo una naturalezza de autodarse las propias reglas y la concentración del sujeto sobre sí mismo. Podríamos hablar de la fe narcisista, que es probable que nos deje indiferentes sobre lo que nos rodea. Es esta la indiferencia

(...) de quien está bien informado, escucha la radio, lee los periódicos o ve programas de televisión, pero lo hace de manera frívola, casi por mera costumbre: estas personas conocen vagamente los dramas que afligen a la humanidad pero no se sienten comprometidas, no viven la compasión. Esta es la actitud de quien sabe, pero tiene la mirada, la mente y la acción dirigida hacia sí mismo. (Francisco, 2016b, párr. 9)

Otras personas, incluso, prefieren no buscar, no informarse, y viven su bienestar y comodidad, sordas al grito de dolor de la humanidad que sufre. 
¿Cómo luchar contra estos ídolos? Las obras de misericordia son un camino viable para la liberación:

Mediante las [obras de misericordia] corporales tocamos la carne de Cristo en los hermanos y hermanas que necesitan ser nutridos, vestidos, alojados, visitados; mientras que las espirituales — aconsejar, enseñar, perdonar, amonestar, rezartocan más directamente nuestra condición de pecadores. Las obras corporales y las espirituales nunca tienen que separarse. Precisamente tocando en el mísero la carne de Jesús crucificado,

el pecador puede recibir como don la conciencia de que él mismo es un pobre mendigo. A través de este camino también los "soberbios", los "poderosos" y los "ricos", de los que habla el Magnificat, tienen la posibilidad de darse cuenta de que son inmerecidamente amados por Cristo crucificado, muerto y resucitado por ellos. Solo en este amor está la respuesta a la sed de felicidad y de amor infinitos que el hombre, engañándose, cree poder colmar con los ídolos del saber, del poder y del poseer. (Francisco, 2016c, párr. 8)

\section{Hacia una Pedagogía de la Misericordia}

En su Mensaje para el Día Mundial de la Paz, el 01 de enero, el papa Francisco solicitó la acción compasiva y misericordiosa de Dios hacia su pueblo. En ese contexto, comentó el pasaje del Éxodo donde el Señor dijo a Moisés: 
He visto la aflicción de mi pueblo que está en Egipto y he oído su clamor a causa de sus opresores; soy consciente, pues, de sus sufrimientos. Así que he bajado para librarlos de manos de los egipcios y sacarlos de aquella tierra a una tierra buena y ancha, a una tierra que mana leche y miel. (Ex 3,7-8)

Es importante tener en cuenta los verbos que describen la actuación de Dios: Él observa, escucha, sabe, baja, libera. Dios no es indiferente. Se puede, entonces, a partir de estos verbos, dibujar las bases para una pedagogía de la misericordia:

\section{- Observar}

La observación exige pararse, detener otras acciones o pensamientos, no ir más allá, dando realidad a la adquirida y conocida. Nos exige, en primer lugar, un acto de liberación de nuestros esquemas, rígidos y cerrados, para abrirnos a los demás. Nos preocupamos por una persona cuando se sabe proteger la trascendencia de otra persona, es decir, cuando se descubre que siempre estuvo esperando detrás de nosotros. Me gusta eso de "llegar tarde". Es una exigencia fenomenológica: poner entre paréntesis nuestras preconcepciones, para recibir y ver lo real por aquello que realmente es. Es el primer paso, pero exige una lucha interna, ejercicio y es un requisito previo para dar el siguiente paso.

\section{- Oír}

Escuchar el grito de la humanidad herida. La simple observación no es suficiente para despertar nuestras interiores entrañas de misericordia. 
Es necesaria la compasión. Desde el punto de vista bíblico, confraternizar no solo significa sufrir con, compartir los mismos sentimientos que la otra persona. Un significado posterior, más profundo, es llevar sobre sí el peso del otro, asumir la responsabilidad. No permanecer indiferentes. Por eso, el papa Francisco recuerda a menudo el tema de la "globalización de la indiferencia": el riesgo de quien cierra los oídos del propio corazón, de quien se hace el sordo a la realidad — no solo a la exterior-, porque la primera indiferencia, la más grave, es aquella hacia Dios. Se trata, para un educador, aunque no es solo su competencia, de escuchar en lo más profundo lo que impulsa a la persona a hacer o decir algo, antes de etiquetarlo, de encuadrarlo en un esquema, especialmente cuando lo que hace nos perturba y crea en nosotros una reacción negativa. Incluso "escuchar" exige un esfuerzo. Por esto, va unido al siguiente verbo: "saber". Este tiene que ser estudiado con el anterior y no por separado.

\section{- Saber}

¡No es suficiente un estudio de laboratorio, no se pueden encontrar las respuestas sentado detrás de un escritorio o un ordenador, quedándonos en la biblioteca! Al padre Bergoglio, cuando se encargaba de la formación de los jóvenes jesuitas en Argentina, se le dio una parroquia cercana. Intentó superar la actitud frecuente de esperar a que los otros, los feligreses, viniesen (actitud pasiva y no demasiado realista). Buscó un mapa del territorio y lo dividió en pequeñas áreas. Entregó cada área a seminaristas voluntarios. ¿Qué tenían que hacer? Ensuciarse los pies... es decir, caminar por las calles y conocer a las personas que vivían en esas calles: se trataba de conocer 
a todas las personas posibles, pobres y los que sufrían, y prestar atención a sus necesidades, evangelizar a los niños. Esto se debía a que los problemas son más complejos, heterogéneos, específicos en comparación con lo que es posible analizar desde un escritorio. Por ejemplo, un problema de la parroquia era que muchas familias solo podían dar a sus hijos una comida. En estos casos, es necesario poner en práctica proyectos. Las primeras iniciativas nos permiten activar las segundas, más específicas, y al mismo tiempo ampliar nuestra percepción de los problemas, ampliar las perspectivas y encaminarnos hacia el camino correcto. Así, los voluntarios organizaron una gran cocina y empezaron a distribuir los alimentos. Bergoglio a menudo daba este consejo: "Estar cerca de los pobres forma el corazón del sacerdote". Para entender completamente la realidad tenemos que pasar de la posición central de calma y paz, y dirigirnos a las zonas periféricas, sin caer en la tentación de "domesticación de las fronteras" (Spadaro, 2013, p. 24), donde, en lugar de ir nosotros hacia aquellas, las traemos a casa para pintarlas un poco y domesticarlas.

\section{- Bajar}

Dios baja sobre su criatura, se acerca, porque sabe que en ciertas situaciones esa criatura no es capaz de hacer más. Se siente satisfecho también con un pequeño resquicio para permitir entrar la gracia de su misericordia que sana. Es un Dios peregrino que sabe, como escribía Don Bosco, que en cada persona, incluso la más difícil, hay un punto accesible al bien, y la tarea de todo educador es encontrar esa cuerda y hacerla vibrar. Es como Jesús con Pedro en el lago de Tiberíades, cuando por tercera vez, preguntando a Pedro si lo amaba, 
se puso al mismo nivel del lenguaje de su discípulo: “¿Me amas?”. Donde no llega el hombre, baja un Dios misericordioso, es suficiente solo dar un paso o al menos desearlo. ¡Bajar entonces es servir! Es lo que el papa Francisco llama la "disciplina de los pies": se refiere al lavado de los pies, Jesús realiza un acto revolucionario, jugando una tarea reservada a los esclavos no judíos, porque era una tarea impura incluso para los esclavos judíos. Es un signo que anuncia: "Estoy a tu servicio" (Francisco, 2013a, párr. 1). "No olvidemos nunca que el verdadero poder es el servicio (...), sólo aquéllos que sirven con amor son capaces de proteger" (Francisco, 2013b, párr. 10). Es necesario bajar, abajarse como Jesús, que vino no para ser servido, sino para servir, bajando hasta la muerte, muerte en la cruz, para salvarnos, para servirnos. En la Iglesia no hay otro camino a seguir. "Para el cristiano ir hacia adelante, progresar, significa abajarse. Si no aprendemos esta regla cristiana, nunca seremos capaces de entender el verdadero mensaje de Jesús sobre el poder” (Francisco, 2013c, párr. 5).

\section{- Rescatar}

Llevar esperanza, hacer sentir la misericordia de Dios no a todos, sino a cada uno, como hace el Buen Pastor. El cristiano que educa nunca puede perder la perspectiva escatológica, la capacidad de orientarse hacia algo mayor, lo que nos trasciende. Es capaz de ver y disfrutar en el momento presente de las semillas de la belleza y la verdad. El amor es visionario y creativo. Es la capacidad de vislumbrar lo que está oculto, lo que aún no se ha hecho presente para rescatarlo. Es capaz, también, de cumplir cuanto es necesario, aunque salga de los modelos ya puestos con anterioridad. No se puede responder a las 
personas con las recetas. Estoy pensando en dos cuadros del pintor belga René Magritte. En el primero, el autor pinta un huevo cerrado en una jaula; en el segundo, un pintor (él mismo), mirando un huevo, pinta en el lienzo un pájaro que inicia a volar. El educador con una mirada de misericordia sabe captar esta belleza presente en cada persona. Sabe poner en cuestión los propios esquemas antes de que estos enjaulen la energía creativa de la persona. ¡Educar es un acto de liberación! Pensad en el trabajo de Paulo Freire y su pedagogía de los oprimidos, que hoy podemos volver a leer como una liberación de la opresión de la indiferencia, de una comunicación de entumecimiento, para retomar el contacto con lo humano. Así, Freire escribió: "La educación auténtica no se hace A hacia $\mathrm{B}$, o A sobre $\mathrm{B}$, sino A con $\mathrm{B}$, con la mediación del mundo. Uno de los malentendidos de una concepción acrítica del humanismo es olvidarse de la situación concreta, existencial, presente, de los propios hombres, debido a la ansiedad de encarnar un modelo ideal de 'hombre bueno"' (Freire, 2005, p. 113).

\section{Conclusión}

Creo que puedo decir unas últimas palabras, por lo que intentaré seguir la trayectoria de una pedagogía de la misericordia: ¡saber arriesgar! Aquí, también, el papa Francisco fue muy claro:

Un maestro que no sabe arriesgar, no sirve para educar. Un padre y una madre que no saben arriesgar, no educan bien a su hijo. ¿Qué significa esto? Enseñar a caminar. ¡Donde hay 
severidad no hay humanismo, y donde no hay humanismo no puede entrar Cristo! ¡Las puertas están cerradas! El drama del cierre comienza en las raíces de la severidad. El verdadero educador ha der ser un maestro del riesgo, pero del riesgo razonable, se entiende. (Francisco, 2015c, párr. 7)

“Dios es creativo, no está encerrado, y por eso nunca es rígido. ¡Dios no es rígido!" (Francisco, 2013d, párr. 9). Ser creativo, para un educador, significa reconocer la necesidad de correr el riesgo de un cambio. Cambiar no para crear necesariamente cosas nuevas o extravagantes, sino para ser fieles. Ser creativos en el Espíritu, con sabiduría, representa la medida del riesgo que podemos tomar para seguir siendo fieles a la realidad, para adaptarnos a las circunstancias en las que anunciar el Evangelio. "Para permanecer con Dios, hay que saber salir, no tener miedo de salir" (Francisco, 2013d, párr. 9).

Solo una pastoral en clave misionera, que abandone el cómodo criterio pastoral de "siempre se ha hecho asi", puede llegar a los corazones de los hombres. Esta es la invitación del papa Francisco en su Exhortación Apostólica Evangelii Gaudium (EG): "Ser audaces y creativos en esta tarea de repensar los objetivos, las estructuras, el estilo y los métodos evangelizadores de las propias comunidades" (EG 33).

Saber arriesgar, además, a sabiendas de que somos falibles. Lo que nos hace fuertes, incluso a los ojos de las personas que educamos o encontramos, es nuestra fragilidad, vulnerabilidad, autenticidad, que no se ocultan detrás de los patrones, papeles, severidad. Porque nuestra fuerza viene del Señor, que no nos pide ser perfectos, sino solo dejarnos amar. Esto es, aceptar la gracia de la misericordia en nosotros para poderla volver a dar, 
igualmente, de manera gratuita. Por esa razón, "Misericordiosos como el Padre" es el lema del Jubileo.

Os dejo con las palabras del compositor norteamericano Leonard Cohen. Él, con una fascinante fuerza poética, nos recuerda en su canción "Anthem" precisamente lo que os acabo de decir:

Tocad las campanas que todavía pueden sonar.

Olvidad vuestra oferta perfecta:

hay una grieta en todo.

Así es como entra la luz. 


\section{Referencias}

Bergoglio, J. M. (2010). Conferencia con ocasión de la XIII Jornada Arquidiocesana de Pastoral Social. Hacia un bicentenario en justicia y solidaridad 2010-2016. Nosotros como ciudadanos, nosotros como pueblo. Recuperado de http://www.arzbaires.org.ar/inicio/homilias/ homilias2010.htm

Francisco. (2013a). Homilía. Santa Misa en la Cena del Señor, realizada en el Centro Penitenciario para Menores Casal del Marmo. 28.03.13. Recuperado de http://w2.vatican.va/content/francesco/es/ homilies/2013/documents/papa-francesco_20130328_coena-domini. html

Francisco. (2013b). Homilía. Santa Misa por la Imposición del palio y entrega del anillo del Pescador en el solemne inicio del Ministerio Petrino del Obispo de Roma. Plaza De San Pedro. Solemnidad de San José. 19.03.13. Recuperado de https://w2.vatican.va/content/francesco/es/ homilies/2013/documents/papa-francesco_20130319_omelia-iniziopontificato.html

Francisco. (2013c). Homilía del Santo Padre en la Casa de Santa Marta. Recuperado de http://www.caminocatolico.org/home/papafrancisco/4981-papa-francisco-en-la-homilia-en-santa-marta2152013-qel-verdadero-poder-es-el-servicio

Francisco. (2013d). Discurso. A los participantes en un Congreso Internacional sobre Catequesis. 27.09.13. Recuperado de https://w2.vatican.va/ 
content/francesco/es/speeches/2013/september/documents/papafrancesco_20130927_pellegrinaggio-catechisti.html

Francisco. (2013e). Exhortación Apostólica Evangelii Gaudium. A los obispos, a los presbíteros y diáconos, a las personas consagradas y a los fieles laicos sobre el anuncio del Evangelio en el mundo actual. 24.11.13. Recuperado de http://w2.vatican.va/content/francesco/es/apost_ exhortations/documents/papa-francesco_esortazione-ap_20131124_ evangelii-gaudium.html

Francisco. (2014). Discurso. Encuentro con la escuela italiana. 10.05.14. Recuperado de https://w2.vatican.va/content/francesco/es/ speeches/2014/may/documents/papa-francesco_20140510_mondodella-scuola.html

Francisco. (2015a). Homilía. Santa Misa de Clausura de la XIV Asamblea General Ordinaria del Sínodo de los Obispos. 25.10.15 Recuperado de http://w2.vatican.va/content/francesco/es/homilies/2015/documents/ papa-francesco_20151025_omelia-chiusura-sinodo-vescovi.html

Francisco. (2015b). Misericordiae Vultus. Bula de Convocación del Jubileo Extraordinario de la Misericordia. 11.04.15. Recuperado de https:// w2.vatican.va/content/francesco/es/apost_letters/documents/papafrancesco_bolla_20150411_misericordiae-vultus.html

Francisco. (2015c). Congreso mundial organizado por la Congregación para la Educación Católica. Recuperado de http://es.radiovaticana. va/news/2015/11/23/preparar_los_corazones_para_que_el_ se\%C3\%B1or_se_manifieste,_el/1188652 
Francisco. (2015d). Carta Encíclica Laudato si. Sobre el cuidado de la casa común. 24.05.15. Recuperado de http://w2.vatican.va/content/ francesco/es/encyclicals/documents/papa-francesco_20150524_ enciclica-laudato-si.html

Francisco. (2016a). El nombre de Dios es misericordia. Una conversación con Andrea Tornielli. Barcelona, España: Editorial Planeta.

Francisco. (2016b). Mensaje. XLIX Jornada Mundial de la Paz: Vence la indiferencia y conquista la paz. 01.01.16. Recuperado de http:// w2.vatican.va/content/francesco/es/messages/peace/documents/papafrancesco_20151208_messaggio-xlix-giornata-mondiale-pace-2016. html

Francisco. (2016c). Mensaje. Cuaresma 2016: "Misericordia quiero y no sacrificio" (Mt 9,13). Las obras de misericordia en el camino jubilar.10.04.15. Recuperado de https://w2.vatican.va/content/ francesco/es/messages/lent/documents/papa-francesco_20151004_ messaggio-quaresima2016.html

Frankl, V. (1991). El hombre en busca de sentido. Barcelona, España: Editorial Herder.

Freire, P. (2005). Pedagogía del oprimido. México D.F., México: Siglo XXI.

Spadaro, A. (2013, 19, 23 y 29 de agosto). Entrevista exclusiva. Papa Francisco: Busquemos ser una Iglesia que encuentra caminos nuevos. Recuperado de http://razonyfe.org/images/stories/Entrevista_al_papa_Francisco. pdf 